The European Physical Journal B manuscript No.

(will be inserted by the editor)

\title{
Selection of dune shapes and velocities. Part 2: A two-dimensional modelling.
}

\author{
Bruno Andreotti ${ }^{1}$ and Philippe Claudin ${ }^{2}$ \\ 1 Laboratoire de Physique Statistique de l'Ecole Normale Supérieure, 24 rue Lhomond, 75231 Paris Cedex 05, France. \\ ${ }^{2}$ Laboratoire des Milieux Désordonnés et Hétérogènes (UMR 7603), 4 place Jussieu - case 86, 75252 Paris Cedex 05, France.
}

October 29, 2018

\begin{abstract}
We present in this paper a simplification of the dune model proposed by Sauermann et al. which keeps the basic mechanisms but allows analytical and parametric studies. Two kinds of purely propagative two dimensional solutions are exhibited: dunes and domes, which, by contrast to the former, do not show avalanche slip face. Their shape and velocity can be predicted as a function of their size. We recover in particular that dune profiles are not scale invariant (small dunes are flatter than the large ones), and that the inverse of the velocity grows almost linearly with the dune size. We furthermore get the existence of a critical mass below which no stable dune exists. However, the linear stability analysis of a flat sand sheet shows that it is unstable at large wavelengths and suggests a mechanism of dune initiation.
\end{abstract}

PACS. 45.70.-n Granular systems - 47.54.+r Pattern selection; pattern formation

\section{Introduction}

The beauty of the crescentic barchan dunes have recently attracted the interest of physicists for a better understanding and modelling of sand transport, as well as ripples and dunes formation and propagation. E. Guyon had this witty remark about them: 'barchans are our drosophila', which means that beyond the scientific and fundamental works on these dunes, we all keep in mind that such studies may lead to potential applications in the fight of saharan countries against sand invasion. One of the first reference work in the field is certainly the famous book of Bagnold which dates back from 1941 [1]. Since then, a great effort of measurement and modelling has been done which we have reviewed in details in the first part of these twin papers.

Our aim here is to discuss and model the selection of two-dimensional dune shape and velocity. For that purpose, we will simplify the model proposed by Sauermann et al. 22,3,, . We will show that although rather severe approximations, we are able to recover their main results, in particular that dune profiles are not scale invariant, and that the inverse of the velocity grows almost linearly with the dune size. Besides, analytical expressions of dome and dune propagative profiles can be obtained, but whose coefficients have to be numerically computed. We furthermore get the existence of a critical mass below which no stable dune exists. The apparition of dunes can however be understood with the linear stability analysis of a flat sand sheet which is unconditionally unstable towards large wavelengths perturbations.
The paper is organized as follows. Section 2 is devoted to the equations of the model. The linear stability of a uniform sand sheet is treated in section 3. In section 14 we simplify further the equations and show what is the general shape of the purely propagative solutions of the model. The specific case of domes and 'actual' dunes are discussed in sections 5 and 6 respectively. At last, we conclude with a discussion of the relevance of these results, and the possible extension of the model to three-dimensional situations and dynamical studies.

\section{Basic equations}

We wish to give a description of the shape and evolution of two-dimensional dunes in terms of two fields: the profile $h(x, t)$ and the volumic sand flux $q(x, t)$ which is the volume of sand transported through an infinite vertical line per unit time. $x$ denotes the horizontal coordinate and $t$ is the time. We are going to write a set of three equations for these quantities in order to include into the model (i) the mass conservation, (ii) the progressive saturation of sand transport and (iii) the feedback of the topography on the sand erosion/deposition processes. Although we shall keep in this paper to two-dimensional situations which correspond to transverse dunes (invariant in the direction perpendicular to the wind), our ultimate goal is of course to be able to describe three dimensional dunes and barchans in particular.

Because barchans in dune fields - so-called 'ergs' organize themselves like gooses or ducks during their migration flights, we named this class of models for purely 


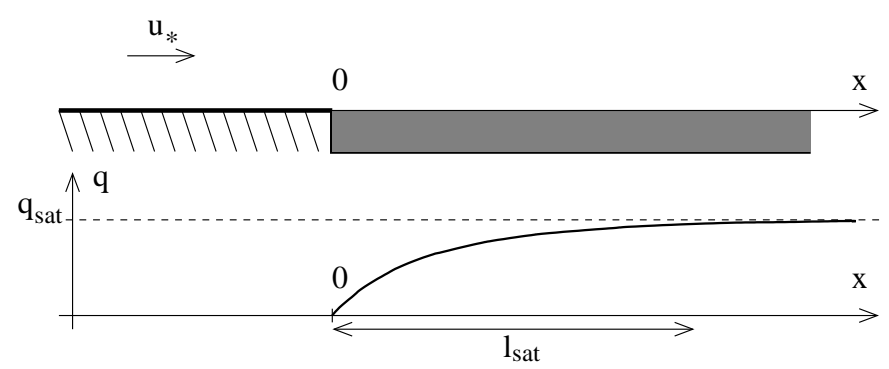

Fig. 1. When the wind is blowing on a patch of sand, the flux of transported sand get saturated after a typical length $l_{\text {sat }}$ which is almost independent of the wind shear velocity $u_{*}-$ see part 1.

graphical reasons the $C_{C}^{C}$ modellings. This denomination includes the approach of Sauermann et al. as well as the different variations and simplifications we derived in this paper from their work. At this stage of the modelling, we are however far from being able to take into account such 'interactions' between dunes which are necessary to explain the $C_{C}^{C}$ spatial organisation, and we shall focus on isolated objects only.

A simple balance calculation shows that the erosion rate $-\partial_{t} h$ is directly related to the divergence of the flux $q$. This gives the common continuity equation:

$$
\partial_{t} h+\partial_{x} q=0 .
$$

The saturation effect of the sand transport has been already evoked in the first part of these twin papers: consider a patch of sand on which the wind is blowing - see figure 1. The flux of transported sand $q$ first increases and, because of the feedback of the grains on the wind velocity profile, get saturated after a typical length $l_{\text {sat }}$. In the first part of the paper, we showed that $l_{\text {sat }}=\xi d \rho_{\text {sand }} / \rho_{\text {air }}(d$ is the grain diameter, $\rho$ the densities and $\xi$ a non dimensional prefactor), i.e. is almost independent of the wind shear velocity $u_{*}-$ slow logarithmic dependencies hidden in $\xi$ only. This phenomenon has been reported and studied by several authors, e.g. [1],5]. The real shape of $q(x)$ is certainly more complicated than the one drawn on figure 1. In particular, oscillating or overshooting features were reported in [1] when $q$ reaches its asymptote. However, what is important for our purpose is only that a saturated value $q_{\text {sat }}$ is reached after a length $l_{\text {sat }}$. This space lag is satisfactorily described by the following equation:

$$
\partial_{x} q=\frac{q_{\text {sat }}-q}{l_{\text {sat }}} .
$$

This charge equation can be seen as a simplification of that proposed by Sauermann et al. in their continuum saltation model [3]. An important remark is that this equation is valid only if some grains are available on the sand bed. On a firm soil indeed, the flux cannot increase to become saturated. As suggested by Peer and Hakim [6], the right hand side of the relation (2) must be therefore multiplied by some matching function which quickly tends to zero when $h$ is decreased below, say, $d$ the grain diameter, and which is equal to unity above this value - the altitude of the firm soil is $h=0$. Then, the equation (2) becomes non linear but no boundary conditions have to be specified at the edges of the sand covered region. For example, if the matching function tends to zero like $h$, the dune will always keep a thin sand sheet at its back. But if it varies as $\sqrt{h}$, the dune will have a finite extension and will join the firm soil with an horizontal tangent. We shall ignore at present these subtleties but keep them in mind to invoke them later when necessary.

Another important remark is that the time scale on which the dune profile $h$ evolves is incomparably larger than that of the sand flux $q$. We then assume that $q$ adapts its profile instantaneously according to equation (2) and makes $h$ change slowly through equation (1). Therefore any term $\partial_{t} q$ is irrelevant in this modelling.

The saturated flux $q_{\text {sat }}$ is uniform for a flat sand bed only. To the first order, the saturated flux $q_{\text {sat }}$ is a function of the local shear stress $\tau=\rho_{a i r} u_{*}^{2}$ which itself depends - non locally - on the topography: basically, bumps and upwind slopes get more eroded than dips and downwind faces. A classical relationship between the saturated flux and the shear velocity that can be recovered with the scaling arguments of the part 1 of the paper is:

$$
q_{\text {sat }} \propto \frac{\rho_{\text {air }}}{\rho_{\text {sand }}} \frac{u_{*}^{3}}{g} .
$$

In principle, such a relationship is valid far from the velocity threshold $u_{t h r}$ under which no sand can be eroded by the wind, i.e. $q_{s a t}=0$ for $u_{*} \leq u_{t h r}$. Refined formulas can be obtained which essentially smooth the step from 0 to the previous asymptotic expression such as that obtained in part 1:

$$
q_{\text {sat }} \propto \frac{\rho_{\text {air }}}{\rho_{\text {sand }}} \frac{u_{*}}{g}\left(u_{*}^{2}-u_{t h r}^{2}\right) .
$$

In the whole range $-u_{t h r}<u_{*}<u_{t h r}, q_{s a t}$ is null so that in practice, $q_{\text {sat }}$ cannot become negative on a dune. This condition will be used in section 5 .

To close the equations, we have to explicit the spatial variations of the turbulent wind velocity due to the dune profile. The simplest model which verifies the basic requirements (see part 1 ) is certainly the perturbative calculation by Jackson and Hunt [7,8]. Neglecting logarithmic scale dependencies, Kroy et al. [4] have extracted the main features of their work by expressing the shear velocity as:

$$
\frac{u_{*}^{2}(x)}{U_{*}^{2}}=1+A \int \frac{d s}{\pi s} \partial_{x} h(x-s)+B \partial_{x} h(x),
$$

where $U_{*}$ is the shear velocity exerted on a flat bed. More precisely, as discussed in details in the part $1, A$ and $B$ in principle depend on the size of the dune $D$ with $\ln D / z_{0}$ factors, where $z_{0}$ is the roughness of the sand surface. For $D$ varying between 20 and $200 m$ and a roughness of order of the grain size, such a logaritmic factor does not change by more than $20 \%$ over the whole range. This justifies the fact that it is reasonable to take constant effective values for the coefficients $A$ and $B$. Several further important remarks must be made on this expression. First, it must be 
noted that the convolution integral acts on $\partial_{x} h$ roughly like a derivative, leading to a term which encodes curvature effects. But this curvature is dimensionless and thus does not depend on the dune size - in other words, this term can be seen as a curvature rescaled by the dune size. It reflects the observation that the wind velocity increases on bumps (negative curvature) and decreases on hollows (positive curvature). Second, it is a non local term, meaning that the shear velocity depends on the whole shape of the dune. Of course sharp variations of the dune profile will also have a strong local effect. At last, the second term simply takes into account slope effects: positive slopes are more eroded than negative ones. Again, this term does not introduce any new lengthscale.

Expression (5) can be used to close up the set of equations as was done by Kroy et al. in [4] who used besides a more sophisticated - and non-linear - charge equation than (2). It is useful to simplify further the equation linking $q_{\text {sat }}$ to $h$ without loosing too much physics, in order to let more analytical developments. The linear expansion of the expression (5) rewritten in terms of $q_{s a t} \propto u_{*}^{3}$ gives:

$$
\frac{q_{s a t}(x)}{Q_{\text {sat }}}=1+\frac{3}{2} A \int \frac{d s}{\pi s} \partial_{x} h(x-s)+\frac{3}{2} B \partial_{x} h(x),
$$

where $Q_{\text {sat }}=q_{\text {sat }}\left(U_{*}\right)$ is the saturated flux on a flat sand bed submitted to a shear velocity $U_{*}$.

Furthermore, we shall use the saturation length $l_{\text {sat }}$ and flux $Q_{\text {sat }}$ to make our variables dimensionless. Thus, for a given wind shear velocity, all relevant scales of the problem are fixed. For instance, $Q_{s a t} / l_{\text {sat }}$ is the velocity scale, $l_{\text {sat }}^{2} / Q_{\text {sat }}$ the time scale, $Q_{\text {sat }} / l_{\text {sat }}^{2}$ the frequency scale, etc. Note that the strength of the wind is completely encoded in these rescalings.

\section{Stability of a flat sand bed}

Before going further in the modelling of dune shape and propagation, we wish to investigate the problem of dune initiation. Indeed, there are two striking field observations. First, no persistent barchan dunes exist smaller than say, $1 \mathrm{~m}$ high, $20 \mathrm{~m}$ large and $20 \mathrm{~m}$ long. Second, any small conical sandpile blown by the wind disappears even when a sand supply is provided. Then, how can barchan dunes appear?

To investigate this problem, we have integrated numerically equations (1,2,6) with $A=6$ and $B=4$. Two initial conditions were tested. First, a small triangular sandpile at the repose angle is prepared on the firm soil (figure 2). It can be seen that it is rapidly eroded and disappears, as observed in the field and in wind tunnel experiments - see part 1. Second, we look at the evolution of a thin sand sheet (figure 3) disturbed by a flat bump. This initial conditions mimics a sand beach on which sand is deposed by water. It can be seen that the bump propagates downwind and induces a strong erosion of the sand bed in front of it. A second bump nucleates from the initial perturbation which itself induces a strong erosion in front of it, and so on. After some time, a series of growing oscillations

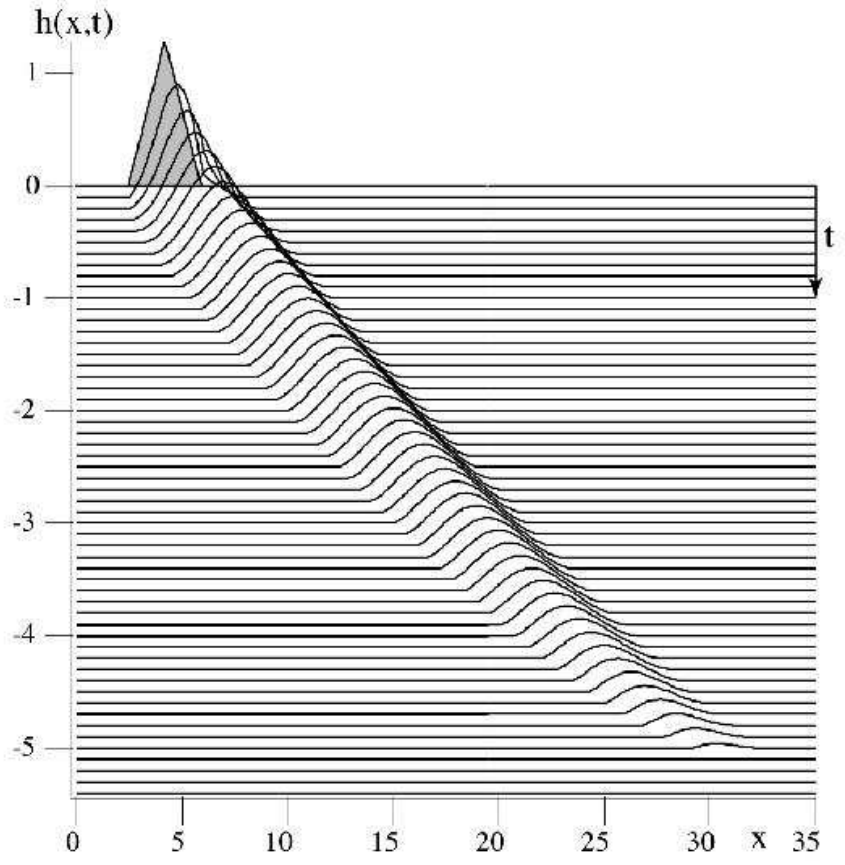

Fig. 2. Numerical integration of equations (1,2,6) for the evolution of a small triangular sandpile on the firm soil, initially $1.3 l_{\text {sat }}$ high and at the repose angle. $h(x, t)$ and $x$ are in units of $l_{\text {sat }}$. The time between two profiles is 0.1 in units of $l_{\text {sat }}^{2} / Q_{\text {sat }}$. For legibility, the profiles are translated vertically from time to time. The grey filled region shows the available amount of sand at $t=0$. The field observation that such a small sandpile disappears when blown by the wind is then recovered in the model.

is generated. The amplification of this phenomenon stops when the oscillations eventually reach the firm soil from which no sand can be eroded, and/or when recirculation bubbles - see section 6- appear and make these bumps interact. As a conclusion, depending on their spatial extension, small sand bumps either disappear or grow and initiate dunes.

It is then instructive to make the linear stability analysis of a flat sand bed. Let us consider an infinite uniform sand bed blown by a uniform wind. The sand flux is everywhere saturated: $q=1$ (in units of $Q_{s a t}$ ). To investigate its stability, we can consider, without loss of generality a small perturbation of the form:

$$
\begin{aligned}
& h(x, t)=H e^{\sigma t-i \omega t+i k x}, \\
& q(x, t)=1+Q e^{\sigma t-i \omega t+i k x},
\end{aligned}
$$

where $Q$ and $H$ are related one to the other by the conservation of matter,

$$
(i \omega-\sigma) H=i k Q .
$$

¿From the relation (6), we get the following expression for the saturated flux: $q_{\text {sat }}=1+3 / 2(|k| A+i k B) h$. Once replaced in the saturation equation, it gives:

$$
\left(1+i k l_{\text {sat }}\right) Q=\frac{3}{2}\left(A|k| l_{\text {sat }}+B i k\right) H .
$$




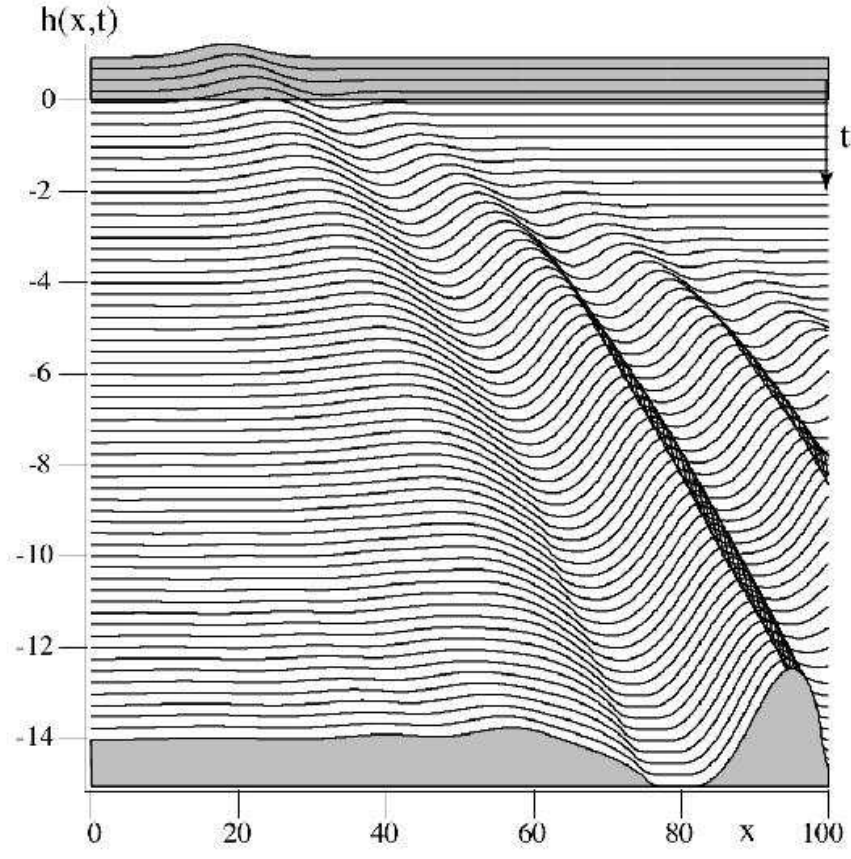

Fig. 3. Numerical integration of equations (1,:2,6) for the evolution of a flat sand sheet of width $l_{\text {sat }}$ over the firm soil disturbed by a small hump. $h(x, t)$ and $x$ are in units of $l_{\text {sat }}$. The time between two profiles is 0.5 in units of $l_{\text {sat }}^{2} / Q_{\text {sat }}$. For legibility, the profiles are translated vertically from time to time. The grey filled region shows the available amount of sand at $t=0$ and at the final time. The perturbation propagates downwind and growing oscillations nucleate from it. The hollow created in front of the initial bump soon reaches the firm soil.

Combining equations (9) and (10) we finally obtain:

$$
\begin{aligned}
& \sigma=\frac{3 k^{2}(B-A|k|)}{2\left(1+k^{2}\right)}, \\
& \omega=\frac{3 k|k|(A+B|k|)}{2\left(1+k^{2}\right)} .
\end{aligned}
$$

Note that a more complicated relation between $q_{s a t}$ and $u_{*}$ would only affect the prefactor, i.e. the time scale, in this calculation.

The growth rate $\sigma$, shown for $A=6$ and $B=4$ on figure 4 (top), is positive for small wavenumbers $(k<$ $B / A)$ and negative for large wavenumbers $(k>B / A)$. A flat sand bed thus exhibits a large wavelength instability which can explain the initiation of dunes. But it is stable towards small wavelength disturbances, so that a small sandpile on a firm soil is quickly eroded.

The fastest growing mode - that which maximises $\sigma$ - is obtained for $3 k+k^{3}=2 B / A$. Neglecting the $k^{3}$ term, we obtain a still good approximation of the most unstable wavelength: $\lambda=2 \pi / k \simeq 3 \pi l_{\text {sat }} A / B$. For $A=6$ and $B=4$ we get $\lambda \simeq 14 l_{\text {sat }}$. For $l_{\text {sat }} \simeq 9 \mathrm{~m}$ we get a wavelength which is reasonable compared to what is observed on transverse dune fields in deserts. Under water $l_{\text {sat }}$ rather scales on the grain size and we have $l_{\text {sat }} \simeq 1 \mathrm{~cm}$ which gives again a good estimation of what has been

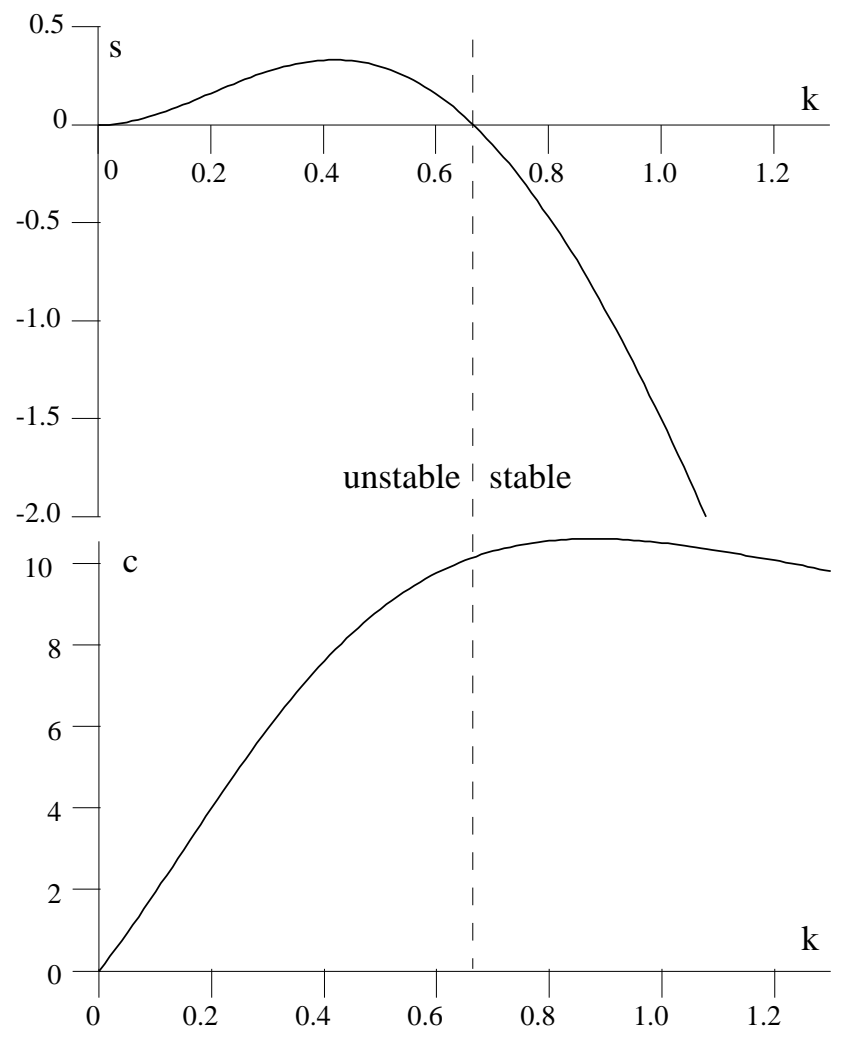

Fig. 4. Results of the linear stability of a uniform sand bed blown by the wind. Top: growth rate $\sigma$, rescaled by $Q_{\text {sat }} / l_{\text {sat }}^{2}$, as a function of the disturbance wave number $k$, rescaled by $1 / l_{\text {sat }}$. The sand bed exhibits a large wavelength instability. Bottom: group velocity $c$ of disturbances, rescaled by $Q_{\text {sat }} / l_{\text {sat }}$, as a function of the disturbance wave number $k$, rescaled by $1 / l_{\text {sat }}$. For small wave numbers, the velocity $c$ increases linearly with $k$ and thus decreases as the inverse of the wavelength.

measured by Betat et al. [9] or Andersen et al. [10] for example.

Finally, it can be seen from equation (12) that disturbances propagate downwind. We can compute the group velocity of these surface waves:

$$
c=\frac{d \omega}{d k}=\frac{3\left[A|k|+B k^{2}\left(3+k^{2}\right)\right]}{2\left(1+k^{2}\right)^{2}} .
$$

$c$ is plotted on figure 1 (bottom) for $A=6$ and $B=4$. For small wavenumbers $k$, the group velocity $c$ increases linearly with $k$. This means that for asymptotically large wavelengths $\lambda$, the propagation speed scales as $Q_{\text {sat }} / \lambda$. We thus recover in the limit of large bumps, the scaling proposed by Bagnold. We also see that the velocity deviates significantly from this law, when the wavelength becomes comparable to the saturation length $l_{\text {sat }}$. This is also the case for actual dunes - see part 1 .

\section{A simple $2 d$ modelling}




\subsection{Simplified equations}

The expression (6) can be kept as it is, mixed with the mass conservation (11) and the charge (2) equations, and treated in Fourier space, but the idea is rather to replace the convolution expression by a simpler anzats. Since it is a 'scaleless and non-local curvature', we can replace it by $-D \partial_{x x} h$, where $D$ is the dune length. $D$ will be determined by the boundary conditions. We then end up with the following set of - linear - equations of our model:

$$
\begin{aligned}
\partial_{t} h+\partial_{x} q & =0, \\
\partial_{x} q & =q_{s a t}-q, \\
q_{s a t} & =1-\alpha D \partial_{x x} h+\beta \partial_{x} h .
\end{aligned}
$$

$\alpha$ and $\beta$ are two of the parameters of the model - a third one, $\mu_{b}$, will be introduced later on. They could be in principle measured independently. The $\alpha$ term has a stabilizing role on small perturbations, while the $\beta$ one make them growing. The rescalings $h \leftarrow \alpha h$ and $t \leftarrow \alpha t$ (with $q$ and $x$ unchanged) shows that only the ratio $\beta / \alpha$ is actually important. As stated in the figure captions, most of the curves of the paper have been plotted with $\alpha=1$. and $\beta=4$. At last, as we already mentioned, the charge equation (15) is valid only if there is some sand to be eroded $h>0$. Note besides that, to be fully consistent, $q_{\text {sat }}$ should remain positive everywhere.

\subsection{Boundary conditions}

Before getting into the process of solving this system of equations, we need to specify what the boundary conditions at the edges of the dune are. This rises several important questions. The first thing to notice is that, when we transformed the relation (6) which links $q_{s a t}$ to the profile $h$ with a convolution term into the simpler expression (16), we have incremented the order of the differential equation. Therefore, such a simplification requires an additional boundary condition than what is needed to integrate equations (6, 14, 15) for example. First order equations like the later need to know the profile $h$ at some position. However, when using the second order equations (14 16), the slope $h^{\prime}$ should be also specified. As we already mentioned, one way to solve this problem is to regularise equation (15) by a non linear factor when $h$ tends to zero which gives conditions on $h(x)$ and $h^{\prime}(x)$ as $x \rightarrow-\infty$, see discussion after equation (2) in section 2.

An alternative is to consider the surface profile - the firm soil as well as the dune - as a whole. This is precisely what is done when the convolution (6) is used. If the slope $h^{\prime}$ was not continuous at the dune edge, in some sense, $h^{\prime \prime}$ would be infinite. Then, from equation (16), the saturated flux would formally tend to $-\infty$ over a region around this edge, i.e. would be forced to be null because it cannot be negative. Thus in this region, only sand deposition is permitted. This means that a discontinuity in the slope at the dune edge, immediately reacts to prevent the motion of this boundary point, which starts moving again only when the profile becomes flat, as shown on figure 5 .

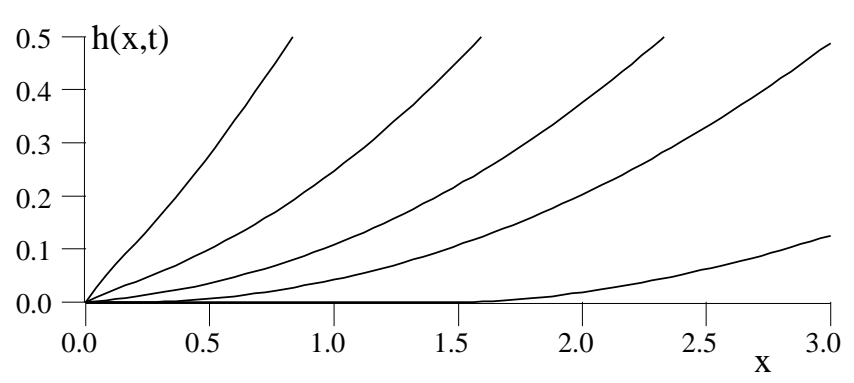

Fig. 5. The numerical integration of equations (6, 14, 15) for an initial sand pile making a finite angle with the firm soil shows that the profile of its upwind left edge quickly evolves to get a horizontal slope which smoothly continue the soil. $h(x, t)$ and $x$ are in units of $l_{\text {sat }}$. The displayed profiles corresponds from left to right to the times $t=0,0.25,0.5,0.75$ and 1.5 in units of $l_{\text {sat }}^{2} / Q_{\text {sat }}$.

Therefore, considering that the wind and thus the saturated flux are not sensitive to the transition from the firm soil to the dune back, gives a 'natural' upwind boundary condition for propagative solutions: the profile $h$ as well as its slope $h^{\prime}$ should vanish at the upwind boundary of the dune. This argument actually also applies at the downwind edge, and we shall use it in the dome and dune next sections.

In the sequel, we are going to look for propagative solutions, i.e. functions of the type $h(x-c t)$ and $q(x-c t)$. We shall see that the explicit form of these functions can be found for given upwind conditions. However, such solutions are parametrized by coefficients such as this length $D$ or the propagative velocity $c$ which must be fixed by the right - downwind - conditions. Two kinds of right boundary conditions will be considered, leading to socalled 'dome dunes', i.e. without avalanche slip face, or 'actual' ones for which a 'recirculation bubble' will be introduced.

\subsection{General form of the propagative solutions}

For functions of $x-c t$, the continuity equation (14) can be easily integrated and gives

$$
q=q_{0}+c h .
$$

We now describe everything in the propagating frame referential, and rename $x$ as the new space coordinate. We look for isolated propagative objects. The point $x=0$ is the beginning of the dune where $h=0 . q_{0}$ is thus the incoming sand flux - the sand supply. In the region $x<0$, no grains are available on the ground: $h=0$ and $q=q_{0}$ everywhere. In the region $x>0$, using the relation between $q$ and $h$ (17) in the charge equation (15) with $q_{\text {sat }}$ given by its expression (16), we get an ordinary differential equation for the dune profile $h$ :

$$
1-\alpha D h^{\prime \prime}+(\beta-c) h^{\prime}-c h-q_{0}=0,
$$




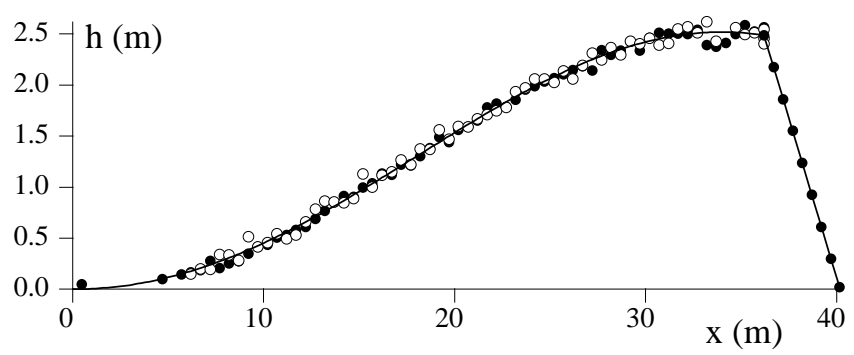

Fig. 6. Longitudinal profile of a barchan dune at Negrita beach, southern Morocco. Black and white dots correspond to independent measurements of the same dune. The solid line correspond to the best fit by a function of the form (19).

where $h^{\prime}$ and $h^{\prime \prime}$ denote the first and second derivatives of the dune profile.

Under the two conditions $h(0)=0$ and $h^{\prime}(0)=0$, the solution of equation (18) is

$$
h(x)=\frac{1-q_{0}}{c}\left[1+\left(\frac{s}{k} \sin (k x)-\cos (k x)\right) e^{s x}\right],
$$

where the coefficients $s$ and $k$ are given by

$$
\begin{aligned}
& s=\frac{\beta-c}{2 \alpha D}, \\
& k=\frac{1}{2 \alpha D} \sqrt{4 c \alpha D-(\beta-c)^{2}} .
\end{aligned}
$$

Figure 6 shows the comparison between the central longitudinal profile of an actual barchan and the theoretical form (19) which is parametrised by $k, s$ and $c$. It can be seen that the shape of the dune as well as the boundary conditions are well captured by the model. In fact, an oscillatory function as (19) does not look like an isolated dune yet. In particular, this $h(x)$ takes negative values. Besides, as already mentioned, the two coefficients $D$ and $c$ are not determined yet. In the next sections we show how it is possible to cut this solution at some point with an adequate right boundary condition in order to get a genuine propagative profile.

\section{Domes}

In this section, we look for so-called 'dome' solutions, i.e. dunes which do not show any avalanche plane. This is possible if the local slope of the dome is everywhere not steeper than some threshold $\mu_{b}$. In the next section, it will become clearer that this threshold corresponds to the slope at which the wind stream lines detach the dune profile, creating a backward wind flow - or a 'recirculation bubble' - which leads to a slip face. As will be explained in the conclusion, these dome solutions may play an important role when extending the present model to three dimensional situations. One of the major results of this section is that the shape of the dome, when it exists, is selected by the value of the incident flux $q_{0}$. Because we

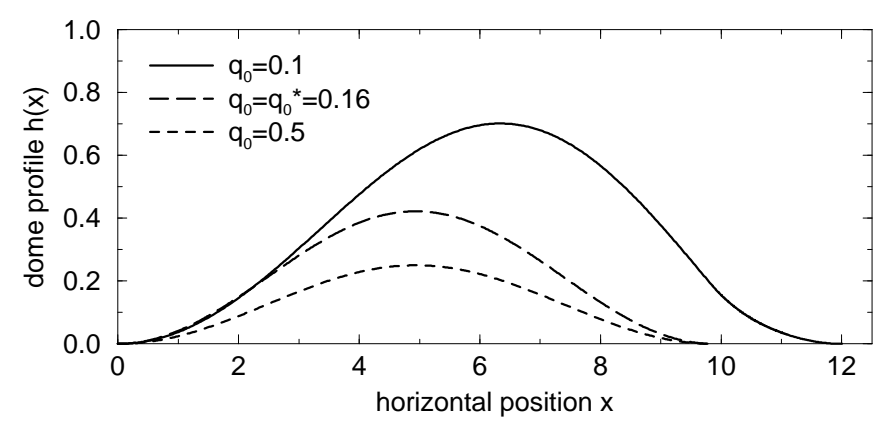

Fig. 7. Shape of the dome solution for different values of the sand flux $q_{0}$. The data have been computed with $\alpha=1$. and $\beta=4$. Note that these domes are actually very flat: for clarity the scale of the vertical axis is much larger than that of the horizontal one.

look for purely propagative solutions, $q_{0}$ of course is also the flux of the sand which leaves the dune.

Let us first take a very simple example. For $c=\beta$ the coefficient $s$ defined in equation (20) vanishes, and the solution (19) reduces to $h(x)=[1-\cos (k x)]\left(1-q_{0}\right) / \beta$, where $k=[\beta /(\alpha D)]^{1 / 2}$. As the general solution (19) was constructed for, $h$ and $h^{\prime}$ vanish at $x=0$, but also at $x=$ $2 \pi / k$. This value sets the length of the dune: $D=4 \pi^{2} \alpha / \beta$. Interestingly, this is precisely the cut-off $\lambda_{c}$ of the linear stability analysis. As discussed in the previous section, this simple solution satisfies the 'natural' downwind boundary conditions $h(D)=0$ and $h^{\prime}(D)=0$. Another important restriction is that on the saturated flux $q_{s a t}$ which must remain positive everywhere. As a matter of fact, there is a minimum value of $q_{0}$ below which $q_{s a t}$ takes negative values and makes this solution inconsistent. It is easy to show that this lower bound for $q_{0}$ reads

$$
q_{0}^{*}=1-\frac{1}{\sqrt{1+\left(\frac{\beta}{2 \pi \alpha}\right)^{2}}} .
$$

For the values $\alpha=1$. and $\beta=4$., we get $q_{0}^{*} \simeq 0.16$. Two such dome profiles for $q_{0}=0.5$ and $q_{0}=q_{0}^{*}$ are displayed on figure 7. At last, note that the steepest slope of this solution is $h_{\min }^{\prime}=-\frac{1-q_{0}}{2 \pi \alpha}$. For $q_{0}=q_{0}^{*}$ and the same numerical values as above, it gives $\left|h_{m i n}^{\prime *}\right| \simeq 0.13$. For any slope threshold $\mu_{b}$ larger than $\left|h_{m i n}^{\prime *}\right|$, the whole set of these solutions such that $q_{0}^{*} \leq q_{0} \leq 1$ is acceptable. For $\mu_{b}$ below this (actually rather small) value however, $q_{0}$ must be larger than $q_{0}^{c}=1 .-2 \pi \alpha \mu_{b}$, and no fully consistent dome solution can be constructed for a smaller incident sand flux. In the sequel, the typical value of $\mu_{b}$ that we shall use will be $\mu_{b}=0.25$.

How can we construct a dome solution for $q_{0}<q_{0}^{*}$ ? Suppose the values of the velocity $c$ and the length $D$ are given, from equation (19) we can compute $h$ and its derivatives as well as $q_{s a t}$. Because $q_{0}$ is smaller than $q_{0}^{*}$, $q_{\text {sat }}$ will reach zero at some position $x=L<D$. Negative values of $q_{\text {sat }}$ are not permitted, and we therefore set it to zero for $x \geq L$. Then, equation (15) is very easy to integrate and gives and exponential branch for the flux 


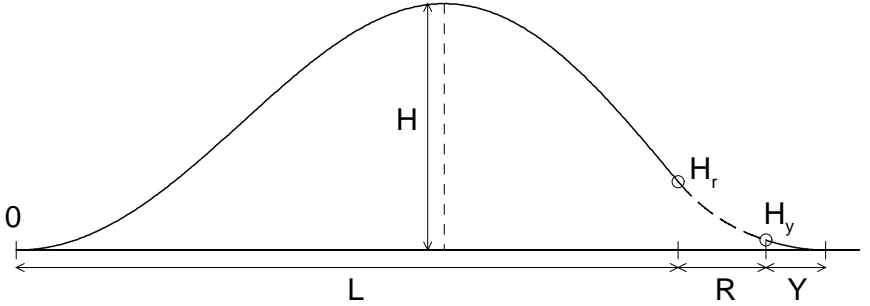

Fig. 8. Definition of the parameters of the dome. $x=0$ is the reference point of the dune. $H$ is its height. For $q_{0}<q_{0}^{*}$, its length $D$ must be cut into three regions of size $L, R$ and $Y$. As explained in the text, in the middle region (dahed line) the saturated flux vanishes and the profile is a branch of exponential which matches with the two parts of the full solution. $H_{r}$ and $H_{y}$ are the heights at the two sides of this central region.

$q(x)$, which, using the linear relation (17) between $q$ and $h$ leads to:

$$
h_{r}(x)=\frac{1}{c}\left[\left(c H_{r}+q_{0}\right) e^{-(x-L)}-q_{0}\right] .
$$

The subscript $r$ is used to avoid any confusion with $h(x)$ given by equation (19), but both are part of the same dome solution. In particular, the dome must be smooth and in this expression $H_{r}=h(L)$ - we shall come back in the next paragraph on the continuity conditions at $x=L$. We cannot end the dome solution with $h_{r}$ for two reasons. First, we argued in the previous section that the dome profile must ends with a horizontal slope, which is not possible with an exponential function at finite distance $D$. Second, if one compute what would be the saturated flux $q_{\text {sat }}^{r}$ calculated from the profile $h_{r}$ with a relation like (16), one sees that it is negative as it should close to $x=L$ (remember that $q_{\text {sat }}$ is set to zero), but crosses zero at some other position $x=L+R<D$. From that point and for larger $x$ we thus need to come back to the original profile $h$. Then, a natural way to end up the dome profile is to use

$$
h_{y}(x)=h(D-x),
$$

which is consistent if $q_{\text {sat }}(-Y)=0$, with $L+R+Y=D$. This choice ensures that both $h(D)$ and $h^{\prime}(D)$ vanish as required. These three regions of the dome solution are illustrated on figure 8 .

Let us now be more explicit about the continuity conditions at the two matching points. At $x=L$, by construction of the relation (23), the dome profile, the sand flux, and the saturated flux are continuous. Because equation (15) holds everywhere, it implies that $\partial_{x} q$, and therefore the slope $h^{\prime}$ are also continuous. By contrast, the curvatures $h^{\prime \prime}(L)$ and $h_{r}^{\prime \prime}(L)$ are different, and therefore $q_{\text {sat }}^{r}(L) \neq q_{\text {sat }}(L)=0$. The position $x=L+R$ is defined by $q_{\text {sat }}^{y}(L+R)=q_{\text {sat }}(-Y)=0$. At this point of course we do not want any step in the dome profile, such that $H_{y} \equiv h_{y}(L+R)$ and $h_{r}(L+R)$ must be equal. Again, because of equation (15) and the fact that $q_{\text {sat }}$ has been built to be continuous at $x=L+R$, the continuity of the profile makes the slope continuous too. However this position is also the point where the pseudo saturated flux $q_{s a t}^{r}$

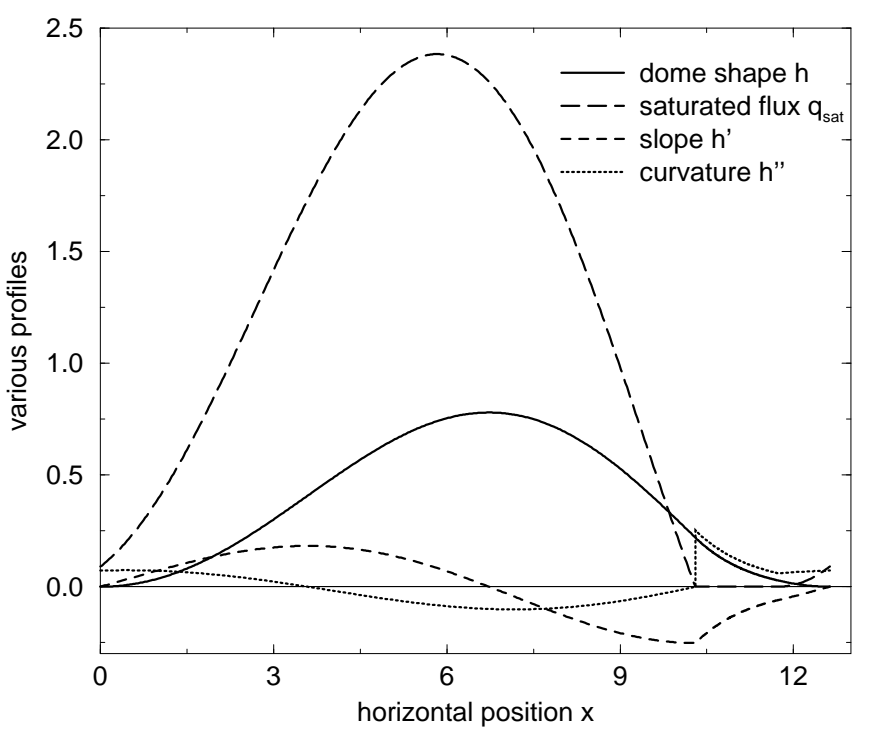

Fig. 9. Dome profile and its derivatives, as well as the saturated sand flux for $q_{0}=0.09$. With this value of the incident sand flux, the dome steepest slope just reaches its minimum permitted value $-\mu_{b}$ at $x=L$. $q_{\text {sat }}$ is strictly zero between $x=L$ and $x=L+R$ and $h$ is a branch of exponential. At $x=L$ the curvature $h^{\prime \prime}$ shows a discontinuity, but all quantities are continuous at $x=L+R$.

crosses zero, such that the curvature of the dome profile is also continuous. All these continuity conditions can be shown on figure 9 .

To sum up, we have in practice four coefficients to determine: $c, L, R$ and $Y(D=L+R+Y)$ with the four non-linear following equations:

$$
\begin{aligned}
q_{\text {sat }}(L) & =0, \\
q_{\text {sat }}(-Y) & =0, \\
h_{r}(L+R) & =h(-Y), \\
q_{\text {sat }}^{r}(L+R) & =0,
\end{aligned}
$$

which can be done numerically. One can find such a solution for any $q_{0}<q_{0}^{*}$. However, to be consistent with the next section, and as already mentioned in the $c=\beta$ case, such a dome is acceptable only if its steepest slope is larger than $-\mu_{b}$. This fixes a lower bound $q_{0}^{c}$ under which there are no solution. With $\alpha=1 ., \beta=4$. and $\mu_{b}=0.25$, we find that $q_{0}^{c} \simeq 0.09$. The solution for this particular value of the incident sand flux is plotted on figure 9, and the dome profile for $q_{0}=0.1$ is compared, on figure 7, with two cases of the $c=\beta$ solutions.

When we have all coefficients of a particular solution, we can compute the mass of the corresponding dome:

$$
M=\int_{0}^{D} d x h(x)
$$

For the $c=\beta$ solutions, this mass can be simply expressed as $M=4 \pi^{2} \alpha\left(1-q_{0}\right) / \beta^{2}$. The interesting point is that the function $M\left(q_{0}\right)$ decreases with its argument, see figure 10 . 


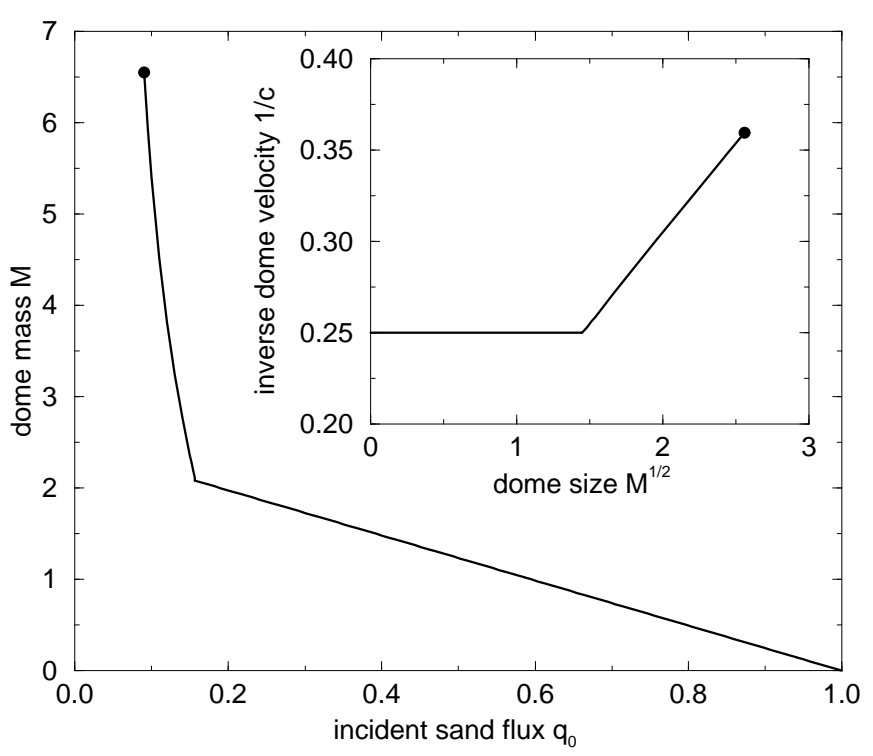

Fig. 10. The main graph shows the mass of a dome selected by an incident flux $q_{0}$. This function is a straight line for $q_{0} \geq q_{0}^{*}$ which corresponds to the $c=\beta$ solutions. The solutions for $q_{0} \leq q_{0}^{*}$ are cut off at $q_{0}=q_{0}^{c}$ where the slope at $x=L$ is equal to the threshold $-\mu_{b}$ (big dot). The important point is that this curve $M\left(q_{0}\right)$ is a decreasing function, which make these domes unstable to a change of $q_{0}$. In the inset, the inverse of the velocity $1 / c$ is plotted against a typical size $M^{1 / 2}$ of the dome. It is strictly constant for $M \leq M\left(q_{0}^{*}\right)$ and then almost straight up to the cut-off value $M_{c}$.

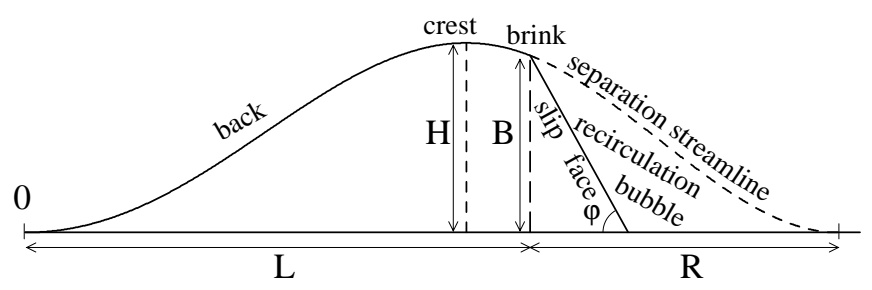

Fig. 11. Definition of the dune and recirculation bubble parameters. $x=0$ is the reference point of the dune. The brink - where the slip face and the recirculation bubble start - is located at $x=L$. The separaration streamline extends the dune profile, starting with the same slope $p$ and curvature $C$. It smoothly reaches the ground at $x=L+R$. We note $H$ the height of the highest point of the dune (crest). $B$ is the dune height at the brink. $H$ and $B$ may coincide or not. We call $\varphi$ the angle of the slip face. For the numerics, we use $\varphi=30^{\circ}$.

Then, take a propagating dome under a flux $q_{0}$, and suppose that, for some reasons, the incident flux is suddenly increased by some small amount $\delta q_{0}$. The shape of the profile is such that the flux of the sand which leaves the dome is exactly $q_{0}$. Then, its mass gets larger. But a larger mass means that the flux of sand which can leave the dome is even smaller than before, which makes the mass of the dome increase again, and so on. In the same way, if the initial flux $q_{0}$ is suddenly decreased, the dome will loose more and more sand because less mass means a higher leaving sand flux. This negative feedback mechanism suggests that domes are unstable objects that may either quickly disappear or reach the point where their slope is steep enough to generate a recirculation bubble and create an avalanche slip face to become an actual dune. One could however observe them, for example under water with periodic boundary conditions [9], which can stabilize such an instability. Then an interesting prediction on the velocity of these domes which could be compared to experiments is shown in the inset of figure 10: it is constant for small domes and $1 / c$ grows almost linearly with their size $M^{1 / 2}$ for larger ones. The dome length $D$ behaves also very much like $1 / c$ as a function of $M^{1 / 2}$.

\section{Dunes}

It is possible to get another type of propagative profiles transverse dunes - with a quite more sophisticated downwind boundary condition, namely a recirculation bubble. It is a common field observation (see part 1) that the wind streamlines on a dune follow exactly the shape of its back profile but separate at the point where the avalanche slip face begins - the brink - and reattach further downwind, as shown on figure 11. This phenomenon creates an eddy in the 'shadow' of the dune, where the wind is much less strong than anywhere else. As a consequence, all the sand eroded on the back is deposited around the top of the slip face which avalanches when the slope becomes too steep. As explained in the first part of these twin papers, it is fortunate that an accurate description of these avalanches is not necessary due to the fact that they do not have any feedback on the back profile of the dune: they simply relax their equilibrium slope $\tan \varphi$. Because no grains can escape the dune from the slip face through the recirculation bubble, the net out flux is zero, which fixes $q_{0}=0$. Note that this is particular to two-dimensional situations that we are focused on: three dimensional barchan dunes loose sand from their horns.

\subsection{The recirculation bubble}

The simplest way to model the feedback of the recirculation bubble on the whole dune has been proposed by Zeman and Jensen 11] and used more recently by Kroy et al. [4]. The idea is to build an envelope of the dune which prolongs the dune profile by the separation streamline see figure 11. To the first order, the wind on the back of the dune is the same as that would have been obtained if the envelope was solid. For example, the convolution integral in equation (5) used to calculate the shear stress on the soil should be applied not to the dune profile but to the dune + bubble envelope.

In our approach, the effect of the recirculation bubble on the wind is simply to modify the total length of the dune $D$ which becomes the dune length $L$ plus the bubble length $R: D=L+R$. The importance of the recirculation bubble becomes very clear: because it makes the apparent 
length of the dune be larger, it increases the erosion at the top of the dune, due to the curvature effect.

We are going to construct a very simple description of the recirculation streamline. Because it extends smoothly the dune shape, it will be possible to link its parameters to the dune ones, and to impose this way what could be called 'non-local right boundary conditions' to the solution expressed by equation (19).

Let us call $h_{b}(x)$ the separation streamline profile. As shown on figure 11, $x=L$ is the point at which this line starts and $x=L+R$ is its reattachment point with the soil. At both matching points, the extension of the dune or the soil by the separation streamline should be smooth:

$$
\begin{aligned}
h_{b}(L) & =h(L) \equiv B, \\
h_{b}^{\prime}(L) & =h^{\prime}(L) \equiv p, \\
h_{b}(L+R) & =0, \\
h_{b}^{\prime}(L+R) & =0 .
\end{aligned}
$$

The recirculation bubble will be essentially governed by one important parameter: a slope that we already named $\mu_{b}$ in the previous section. It corresponds to the maximum angle that the wind streamlines can follow behind an obstacle. In other words, there is a flow separation if the slope is locally steeper than $-\mu_{b}$. It is natural to impose that the steepest slope of the separation streamline should then be $-\mu_{b}$ :

$$
h_{b}^{\prime}\left(x_{b}\right)=-\mu_{b} \quad \text { with } \quad h_{b}^{\prime \prime}\left(x_{b}\right)=0 .
$$

As a matter of fact, this steepest slope should be in principle less than or equal to $-\mu_{b}$ otherwise this would mean that the streamlines actually detach at a higher threshold value. Besides, the bubble initially appears exactly at this slope on critical domes. The relation (34) can be then taken as the simplest self consistent slope condition.

Because the wind velocity profile is scale invariant, a further property is that the length $R$ of the recirculation bubble should scale on the dune size and no new length scale should be introduced by the bubble. Therefore, the curvature at $x=L$ must also be continuous:

$$
h_{b}^{\prime \prime}(L)=h^{\prime \prime}(L) \equiv C .
$$

Such a condition is also naturally observed when numerically integrating equations (6, 14, 15) - i.e. with the convolution term of equation (6) rather than the simplified relation (16). Furthermore, we noticed that the stability of the numerical scheme is very sensitive to the way the slope is computed at $x=L$ : the only choice which does not create any instability is that which uses a formula which mixes the dune and bubble profiles $h^{\prime}(L)=$ $\left[h_{b}(L+\Delta x)-h(L)\right] / \Delta x$, where $\Delta x$ is the discretisation step.

In the absence of available data or models on flow separation behind a dune, the explicit form of the bubble profile we chose is the simplest that satisfies the requirements written above, namely a polynomial of $3^{\text {rd }}$ degree:

$$
h_{b}=B+p(x-L)+\frac{1}{2} C(x-L)^{2}+\frac{1}{6} G(x-L)^{3} .
$$

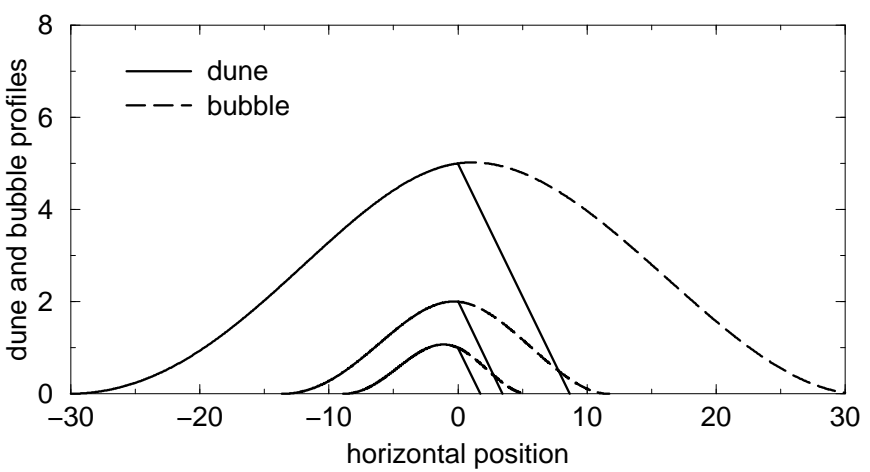

Fig. 12. Dunes of different masses and corresponding recirculation bubbles. From small to large, the masses are $M=6.05$, 16.6 and 88.7. The other parameters are $\alpha=1$., $\beta=4$. and $\mu_{b}=0.25$. For clarity, all these profiles have been shifted in order to get all brink positions at $x=0$.

Such a choice was also that of Kroy et al. in [4]. Using the conditions (30-35), $G, C$ and $R$ can be computed as functions of the slope $p$ and the height $B$ at the brink:

$$
\begin{aligned}
G & =\frac{9 \mu_{b}}{R^{2}}\left(1-\frac{2 B}{3 R \mu_{b}} \sqrt{1-\frac{4 B}{3 R \mu_{b}}}\right) \\
C & =-R G+\sqrt{2 G \mu_{b}} \\
p & =-\mu_{b}+\frac{C^{2}}{2 G}
\end{aligned}
$$

We have checked that other similar choices for the parametrization of the separation streamline do not change the qualitative conclusions that are going to be presented in the next subsections. In a general way, the recirculation bubble conditions can be expressed as $R / B=f_{1}(p)$ and $C B=f_{2}(p)$, where the functions $f_{1}$ and $f_{2}$ encode the particular choice of the streamline separation profile $h_{b}$.

These two relations put together with the explicit expression of the dune profile $(19)$ and the differential equation that it verifies (18), where $D$ has been set to the whole dune + bubble length $D=L+R$, let one to get three relations which link together the four parameters $c$, $L, R$ and $B$. Instead of plotting, say, the three first with respect to the last one, we rather chose to express, as we did for the domes in the previous section, all of them as functions of the total mass $M$ of the dune:

$$
M=\int_{0}^{L} d x h(x)+\frac{B^{2}}{2 \tan \varphi} .
$$

We then get a continuous set of dune solution, from very large values of the mass, down to some cut-off value $M_{c}$ below which no stable recirculation bubble can be constructed - see below.

\subsection{Profiles, dimensions and velocities}

Let us fix a value of the mass of sand $M$ available to construct a dune. As for the domes, even for the simple choice 


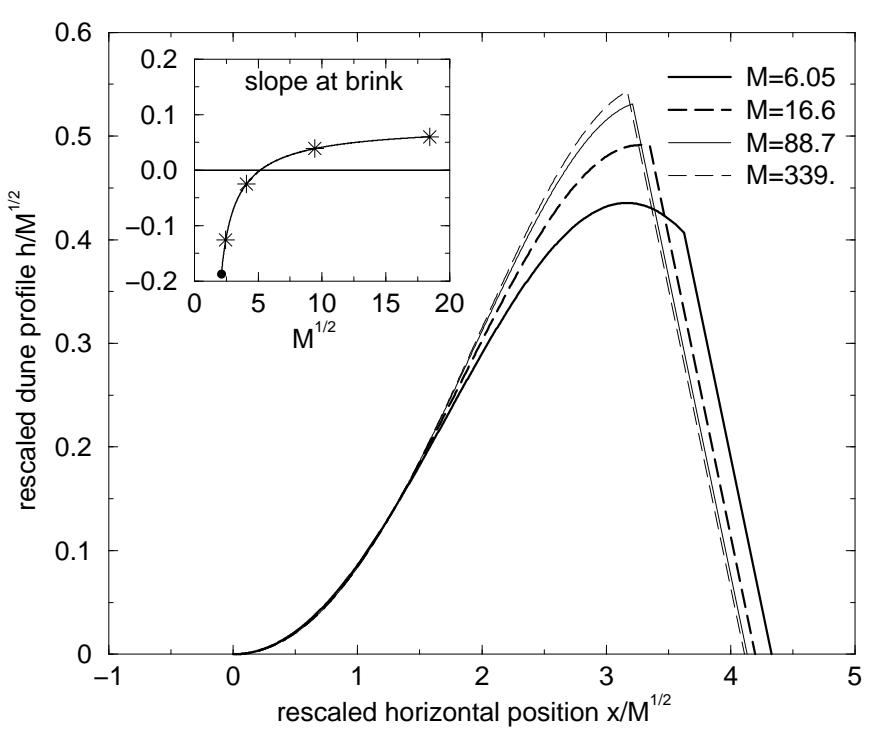

Fig. 13. Rescaled profiles of dunes of different sizes. The data have been computed with $\alpha=1$., $\beta=4$. and $\mu_{b}=0.25$. Lengths have been rescaled by the square roots of the masses of the dunes. These profiles are not scale invariant: as shown in the inset, the slope $p$ just before the brink is negative for small dunes and positive for large ones, such that depending on the dune size, the crest does or does not coincide with the brink. The four stars represent the four dune profiles.

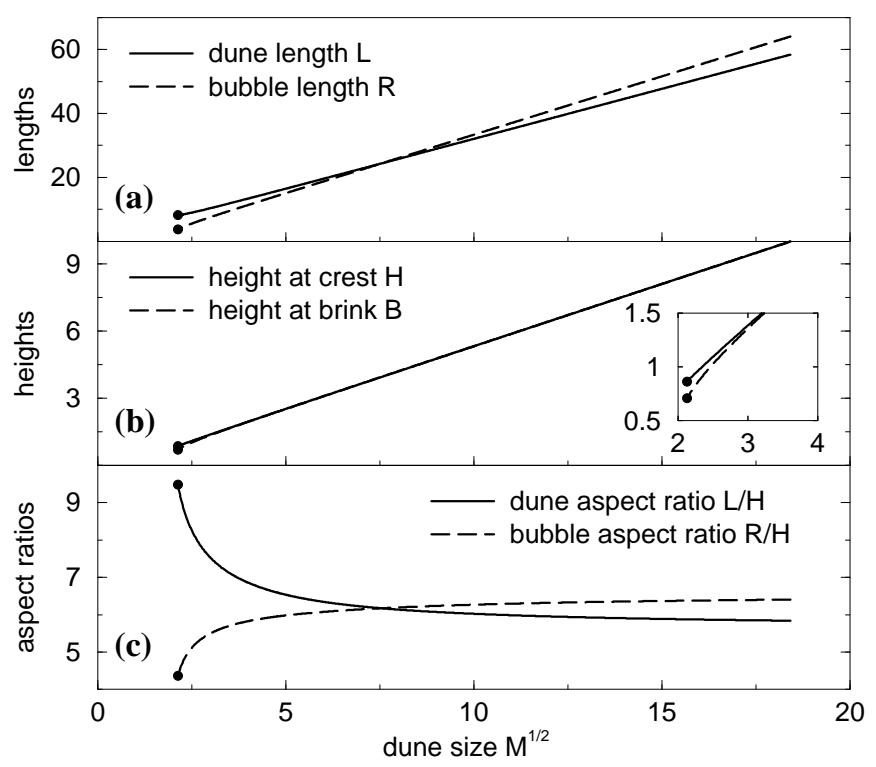

Fig. 14. Scaling of the lengths $L, R$ (a) and $H, B$ (b) with the dune size $M^{1 / 2}$. As a first approximation, these plots are almost straight lines. No recirculation bubble - and therefore no dune solution - can be constructed below some cut-off value (big dot). The inset on graph (b) is a zoom around this cutoff scale. Graph (c) shows that the dune and bubble aspect ratios are not constant: large dunes are more compact with a proportionally larger bubble than small ones. The data have been computed with $\alpha=1$., $\beta=4$. and $\mu_{b}=0.25$.

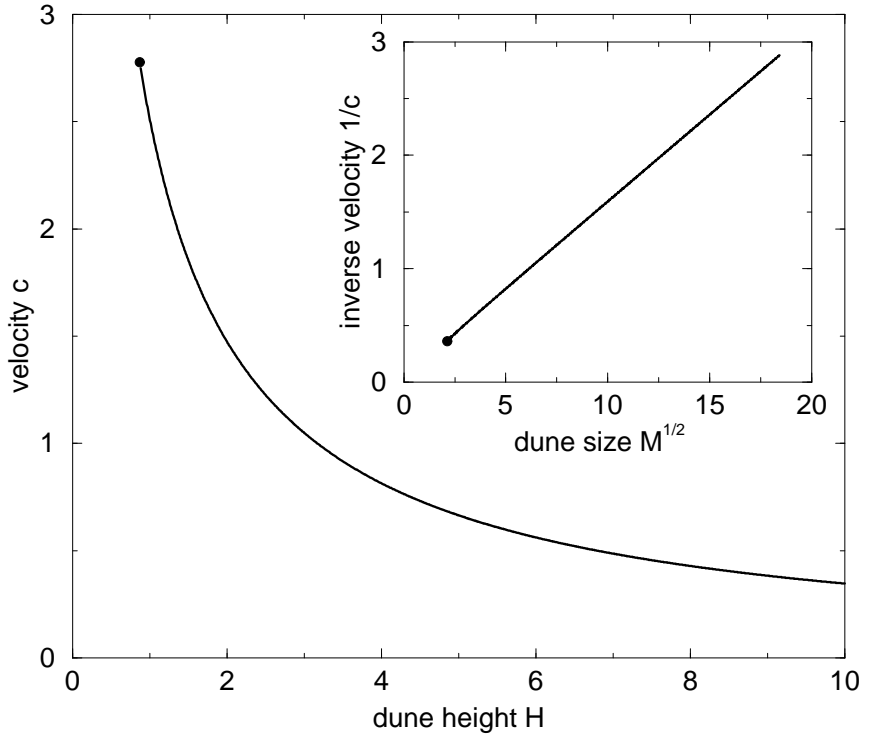

Fig. 15. Propagation velocity $c$ of the dune as a function of its height $H$. As shown in the inset, with a very good precision, $1 / c \sim a M^{1 / 2}+b$ down to some cut-off value. These data have been computed with $\alpha=1$., $\beta=4$. and $\mu_{b}=0.25$.

of the recirculation bubble profile (36), a numerical resolution is required at this point to get the corresponding values of the parameters $c, L, R$ and $B$. Three examples of such solutions are plotted on figure 12. As evidenced on figure 13, when rescaled by a typical dimension of the dune - here the square root of the mass -, the different profiles do not collapse on a single curve. In particular the slope at the brink $p=h^{\prime}(L)$ varies with respect to $M$, and even changes its sign for this choice of the parameters $\alpha$, $\beta$ and $\mu_{b}$. We get $p<0$ for the smallest dune, while $p>0$ for the largest one.

The inset of figure 13, as well as the curves of figures 14 and 15, which all show the dune and bubble features (lengths, slope, aspect ratios and velocity) as a function of the mass of the dune $M$, are all cut off at some value $M_{c}$ (big dot). This point corresponds to the smallest solution that can be constructed. Technically, one can see that the equations (37-39) which link bubble and dune parameters loose their sense when the quantity $3 R \mu_{b}-2 B$ becomes negative. This point precisely gives the cut-off mass under which no bubble, and therefore no dune solution can be constructed. It is important to notice that this smallest dune has an avalanche slip face of finite size. Another choice for the function $h_{b}(x)$ would of course have given different values for $M_{c}$ as a function of the parameters of the model. But physically, this cut-off scale corresponds to the fact that such a recirculation bubble must have a minimum spatial extension to accommodate all continuity constrains. Not surprisingly, this extension is of the order of unity, that is to say of the order of $l_{\text {sat }}$ in non rescaled length units - which is the only length scale of our system. At last, it must be mentioned that the parameters of this smallest dune are pretty close to that of the largest dome, 


\begin{tabular}{|c|c|c|c|}
\hline & $\mathrm{M}$ & $\mathrm{c}$ & $\mathrm{H}$ \\
\hline Smallest dune & 4.51 & 2.78 & 0.86 \\
\hline Largest dome & 6.55 & 2.78 & 0.78 \\
\hline
\end{tabular}

Table 1. Mass, velocity and height of the smallest dune solution that one can construct, compared to that of the largest dome for which the steepest slope is equal to $-\mu_{b}$. Consistently, these data are pretty close to each other. These numerical values have been computed with $\alpha=1$., $\beta=4$. and $\mu_{b}=0.25$.

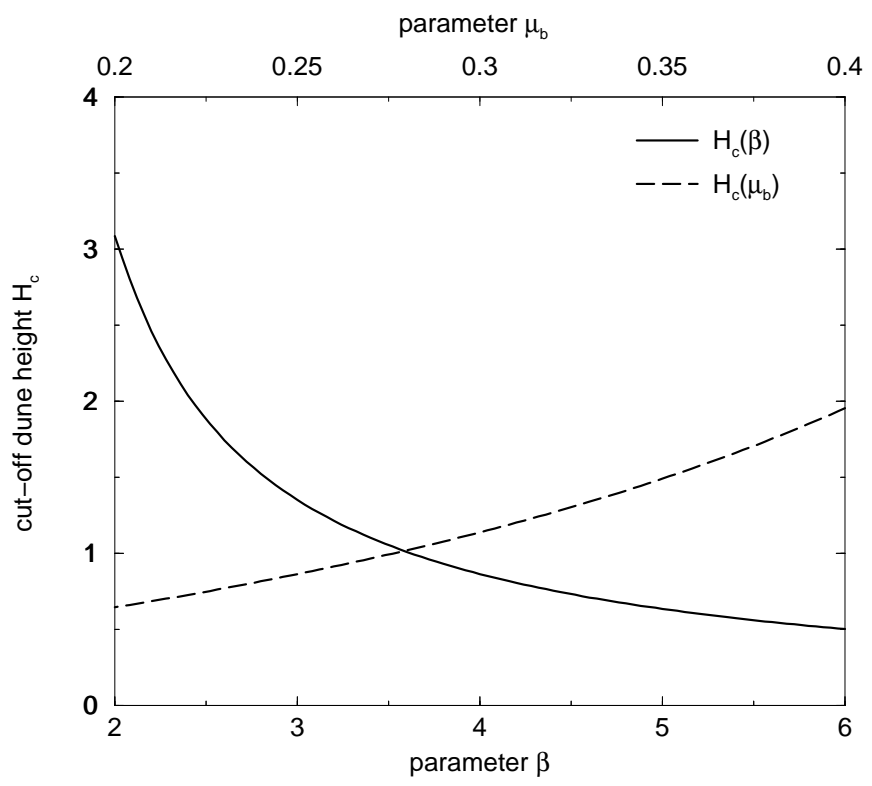

Fig. 16. Variation of the minimal dune height $H_{c}$ with the parameters $\beta$ and $\mu_{b}$. To plot theses curves, we took $\alpha=1$. Besides, the solid line has been obtained with $\mu_{b}=0.25$, and the dashed one with $\beta=4$.

i.e. the dome for which the steepest slope is precisely equal to $-\mu_{b}$, see table 1 .

All results of figures 14 and 15 are remarkably simple and resemble very much field observations: the lengths $L$, $R, H$ and $B$ are almost straight lines as a function of the dune size $M^{1 / 2}$. Similarly, $1 / c \sim a M^{1 / 2}+b$ with a very good precision. At last, it must be noted that the dune and bubble aspect ratios are not constant: large dunes are more compact with a proportionally larger bubble than small ones.

\subsection{Parametric study}

To complete the results of the previous subsection, we present here a parametric study of few quantities, namely the minimal dune height $H_{c}$ and the dune slope at the brink of asymptotically large dunes $(M \rightarrow \infty)$. The parameter $\alpha$ is kept to unity, but $\beta$ has been varied between 2 and 6 , and $\mu_{b}$ from 0.2 to 0.4 .

Figure 16 shows that the variation of $H_{c}$ as a function of the parameters of the model is not very strong.

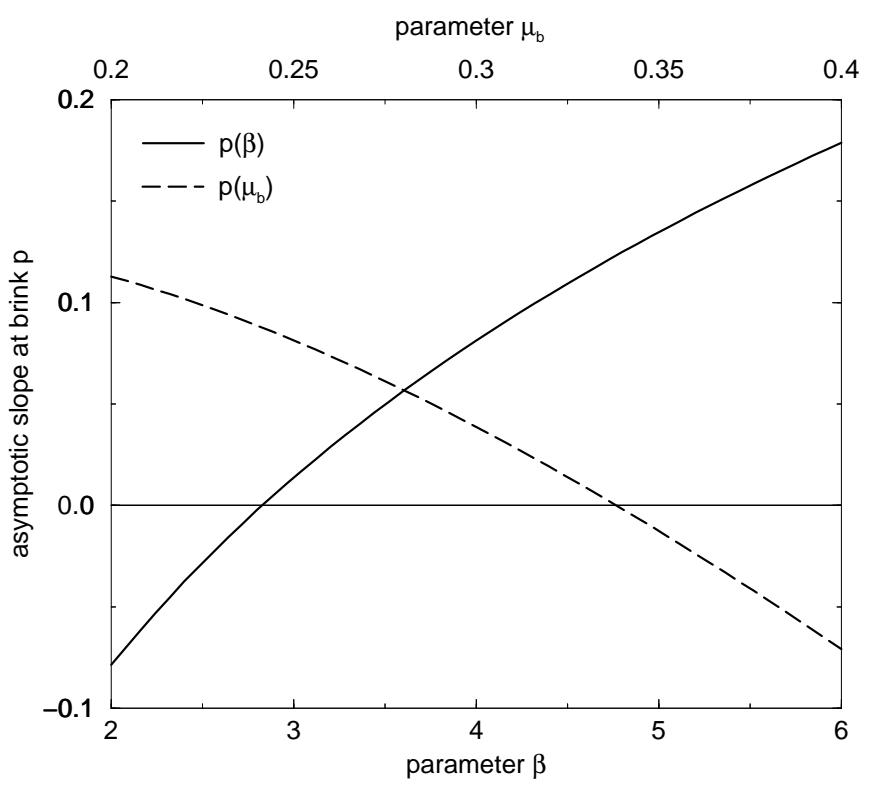

Fig. 17. Variation of the asymptotic $(M \rightarrow \infty)$ slope at the brink $p$ with the parameters $\beta$ and $\mu_{b}$. To plot theses curves, we took $\alpha=1$. Besides, the solid line has been obtained with $\mu_{b}=0.25$, and the dashed one with $\beta=4$. Interestingly, $p$ remains negative for small $\beta$ or large $\mu_{b}$.

Similarly, the aspect ratio of very large dunes is always of the same order of magnitude. The most interesting fact is perhaps that for small values of $\beta$, or for large values of $\mu_{b}$, the slope at the brink of very large dunes can remain negative - see figure 17. In other words, the brink and the crest can be always distinct, even for asymptotically large dunes.

One can understand intuitively the variations of $H_{c}$ and $p$ with $\beta$ and $\mu_{b}$. Increasing $\beta$ gives more strength to the destabilizing process, which lets small dunes appear at lower critical scale (smaller $H_{c}$ ) and makes large dunes more bumpy (larger $p$ ). If $\mu_{b}$ gets larger, the situation is reversed.

\section{Conclusion}

We have shown in this paper how, inspired from the work of Sauermann et al., one can build a simpler two dimensional model for the formation and the propagation of dunes. This modelling is based on two main variables: the dune profile $h$ and the volumic sand flux $q$. It includes three effects: (i) the mass conservation, (ii) the space lag over a length $l_{\text {sat }}$ for the sand flux to become saturated at some value $q_{\text {sat }}$, and (iii) the feedback of the profile on the saturated flux. In this third phenomenological equation, erosion and deposition processes are the result of the competition between two antagonist mechanisms: a stabilizing non local curvature term $(\alpha)$ and a destabilizing slope one $(\beta)$ which breaks the upwind-downwind symmetry. 
Two kinds of solutions have been found: so-called 'domes' which do not show any avalanche slip face, and 'real' dunes for which a downwind recirculation bubble has been introduced. We were able to predict an analytical form for their propagative profiles, but whose coefficients have to be computed numerically.

The results of the model resemble very much field observations. We found for example that, due to this length scale $l_{\text {sat }}$, the dune profiles are not scale invariant: small dunes are flatter than large ones. Another point is that the inverse of the propagative velocity $c$ is, to a very good precision, almost linear with the size of the dune. This is consistent with Bagnold's argument that $c \simeq q_{\text {sat }} / H$ for a dune of height $H$. In fact, this relation overestimates the velocity of small dunes for which the sand flux may not be already saturated at the crest and also because, compared to large dunes, the value of $q_{\text {sat }}$ is reduced due to a smaller curvature.

An important point discussed all through the paper was the issue of the boundary conditions. In particular, an important physical input was the 'recirculation bubble' behind the dune. This bubble makes the dune effectively look larger to the wind, and, due to the non local term in the relation between the saturated flux and the dune profile, has a stabilizing role. In fact, very little is known and well established about this recirculation bubble, but most of the dune features (position of the slip face, cutoff size, etc.) precisely depend on fine interactions and feedbacks between the dune and the bubble. More studies on this point are then urgently needed.

Another central result of the paper is the existence of this cut-off scale just mentioned, below which no dune can form. It corresponds to the fact that the bubble must have a minimum spatial extension to accommodate all continuity constrains. Not surprisingly, this scale is of order of $l_{\text {sat }}$. This result then rises the question of dune initiation and formation. The two scenari usually proposed by geophysicists for the formation of dunes are the following: first possibility, a small bump (of the size of ripples) grows continuously and forms a dune; second one, the sand accumulates on a solid obstacle like a rock or a bush and, when the size of the accumulation becomes larger than the obstacle, a dune forms and starts propagating downwind. However, observations show that ripples are stable and no structures between dunes and ripples can be seen. Similarly, rocks and bushes creates lee dunes of the size of the obstacle which remain anchored to the obstacle. An alternative explanation can be proposed, following the results of the stability analysis of the equations of the model, as well as that of the dome solutions. We found that large wavelengths perturbations get amplified, and that the dome profiles, selected by their incident flux $q_{0}$, are unstable to changes of that flux. Then, a possibility is that first domes form with a small height but directly with large length and width, and second that these domes progressively become more and more compact, and eventually reach the point where their slope is steep enough to generate a bubble and create an avalanche slip face to become an actual dune.
Several extensions to the present work can be thought of. First, we would like to go beyond the calculation of purely propagative solutions, and study the full dynamics of a given dune profile. In particular, as just said, an important point is the evolution of the dome solutions when submitted to incident sand flux variations. A second point is to go from a $2 \mathrm{~d}$ description - transverse dunes to real three dimensional situations. The idea is to 'cut a barchan into longitudinal $2 \mathrm{~d}$ slices'. As a matter of fact, a barchan slice close to the center of the dune looks like our dune solution, while a slice made at the edges where the horns are present rather have a dome shape. Suppose these slices are completely decoupled. Because the small ones go faster than the large ones, an initial conical sandpile will soon get a crescentic shape. However, when equilibrium is reached, all the slices should move at the same velocity. There should thus be a coupling between them, namely a lateral sand flux from the centre towards the horns. When the flux is saturated at the crest the velocity at the crest is $c=\left(q_{s a t}-q_{0}\right) / H$. This suggests than an equilibrium can indeed be achieved if $q_{0}$ increases in the small slices. Eventually the 3D dune slip face will be the sum of the contributions of all 2D slices whose brinks depend on their heights. Note that this scenario is consistent with the field observation that barchan horns are more elongated at strong winds which make the lateral sand flux less important, and consequently slices less coupled.

Finally, quantitative comparisons between experimental dune profiles and our theoretical predictions will be performed. This idea is to use barchan longitudinal slices as that shown on figure 6, but also sand structures under water, such as those obtained by Betat et al. [9] or Andersen et al. [10] for which, in principle, this model should be also valid.

\section{Acknowledgments}

We are grateful to Gerd Sauermann and Klaus Kroy for precise explanations of all the details of their modelling. The measurements of the dune profiles (figure 6) were performed by B. Andreotti, S. Douady, P. Hersen and L. Quartier. We wish to thank Stéphane Douady, Christof Krülle and Brad Murray for useful discussions.

\section{References}

1. R.A. Bagnold, The physics of blown sand and desert dunes, Chapman and Hall, London (1941).

2. Sauermann G., PhD Thesis, Stuttgart University, edited by Logos Verlag (Berlin) (2001).

3. G. Sauermann, K. Kroy and H.J. Herrmann, Phys. Rev. E 64, 031305 (2001).

4. K. Kroy, G. Sauermann and H.J. Herrmann, A minimal model for sand dunes, submitted to Phys. Rev. Lett., cond-mat/0101380 (2001).

5. R.S. Anderson and P.K. Haff, Science 241, 820-823 (1988).

6. B. Peer and V. Hakim, private communication.

7. P.S. Jackson and J.C.R. Hunt, Quart. J. R. Met. Soc. 101, 929-955 (1975). 
8. W.S. Weng, J.C.R. Hunt, D.J. Carruthers, A. Warren, G.F.S. Wiggs, I. Livingstone and I. Castro, Acta Mechanica 2, 1-22 (1991).

9. A. Betat, C.A. Krülle, V. Frette and I. Rehberg, Longtime behaviour of sand ripples induced by water shear flow, submitted to Euro. Phys. J. E.

10. K. Andersen, P. Gondret, M. Rabaud and T. Loiseleux, private communication.

11. N-O. Jensen and O. Zeman, in International workshop on the physics of blown sand, Barndorff-Nielsen, Moller, Rasmussen and Willets editors, University of Aarhus, 351-368 (1985). 